\title{
Characterizing nanoscale scanning probes using electron microscopy: A novel fixture and a practical guide
}

\author{
Tevis D. B. Jacobs, ${ }^{1, a)}$ Graham E. Wabiszewski, ${ }^{2}$ Alexander J. Goodman, ${ }^{2}$ \\ and Robert W. Carpick ${ }^{2, b)}$ \\ ${ }^{1}$ Department of Mechanical Engineering and Materials Science, University of Pittsburgh, 3700 O'Hara St., \\ Pittsburgh, Pennsylvania 15208, USA \\ ${ }^{2}$ Department of Mechanical Engineering and Applied Mechanics, University of Pennsylvania, 220 S. 33rd St., \\ Philadelphia, Pennsylvania 19104, USA
}

(Received 1 October 2015; accepted 23 November 2015; published online 20 January 2016)

\begin{abstract}
The nanoscale geometry of probe tips used for atomic force microscopy (AFM) measurements determines the lateral resolution, contributes to the strength of the tip-surface interaction, and can be a significant source of uncertainty in the quantitative analysis of results. While inverse imaging of the probe tip has been used successfully to determine probe tip geometry, direct observation of the tip profile using electron microscopy (EM) confers several advantages: it provides direct (rather than indirect) imaging, requires fewer algorithmic parameters, and does not require bringing the tip into contact with a sample. In the past, EM-based observation of the probe tip has been achieved using ad hoc mounting methods that are constrained by low throughput, the risk of contamination, and repeatability issues. We report on a probe fixture designed for use in a commercial transmission electron microscope that enables repeatable mounting of multiple AFM probes as well as a reference grid for beam alignment. This communication describes the design, fabrication, and advantages of this probe fixture, including full technical drawings for machining. Further, best practices are discussed for repeatable, non-destructive probe imaging. Finally, examples of the fixture's use are described, including characterization of common commercial AFM probes in their out-of-the-box condition. (C) 2016 AIP Publishing LLC. [http://dx.doi.org/10.1063/1.4937810]
\end{abstract}

\section{INTRODUCTION: THE IMPORTANCE OF ACCURATE DETERMINATION OF TIP SHAPE IN SCANNING PROBE MICROSCOPY (SPM)}

The widespread use of SPM and its variants has enabled detailed characterization of the physical, electrical, and chemical interactions of nanoscale interfaces. Quantitative SPM data analysis often requires knowledge of the geometry of the nanoscale tip used to interact with a sample's surface, since measurements can depend sensitively on the tip size and shape. Examples include determining the work of adhesion using contact mechanics models to analyze atomic force microscopy (AFM) pull-off force measurements; ${ }^{1-4}$ measuring mechanical properties such as elastic moduli and hardness with AFM-based nanoindentation;, ${ }^{5,6}$ extracting the contact stiffness from dynamic AFM measurements; ${ }^{7,8}$ and characterizing the interfacial shear strength (friction). ${ }^{9,10}$ Typically, contact mechanics models assume a tip shape that is a hemisphere, paraboloid, or another simple analytical function $^{11}$ with a geometric parameter like a radius of curvature; the resulting mechanical parameters of contact are compactly described by analytical closed-form equations. Uncertainty in the probe tip shape or radius has been identified as one of the primary sources of error when determining the quality of mechanical interaction between the tip and the countersurface. ${ }^{11-13}$

\footnotetext{
a)Email: tjacobs@pitt.edu

b)Email: carpick@seas.upenn.edu
}

Other scanning probe techniques also require knowledge of the tip shape and of changes that occur with use. For instance, a detailed analysis of piezoresponse force microscopy, ${ }^{14}$ which assumed a spherical probe tip apex and Hertzian contact mechanics, showed that the tip radius directly determines piezoelectric surface response. Another example is provided by an analysis of roughness of the AFM probe tips themselves, ${ }^{13}$ where it was demonstrated that sub-nanometer-scale roughness of the tip can cause orderof-magnitude changes in the measured work of adhesion, with significant deviations from the value predicted when a smooth paraboloidal shape is assumed. Even many SPM measurements that do not require knowledge of the tip shape and size would be significantly more powerful if the tip shape were accurately known. For instance, conductive AFM (C-AFM) and scanning spreading resistance microscopy (SSRM) can be performed on a surface to determine relative conductivity of various regions. However, if the detailed tip shape were known, the effect of insulating surface filmssuch as oxides, passivating species, or contaminants-could be accurately determined, as discussed in a case study in Section V C. In the present paper, we refer to the sharp tips used for scanning probe microscopy as atomic force microscopy tips; however, the results are applicable to all techniques within the SPM umbrella.

For all of the above purposes, it is problematic to trust manufacturers' estimates of the tip shape, or even to assume small variations of the tip shape within a single commercial package of probes. It has been shown, for example, by Sedin 
and Rowlen, ${ }^{15}$ that an AFM tip with a radius specified to be $<10 \mathrm{~nm}$ in fact had a $>50 \mathrm{~nm}$ flat punch apex, resulting in significant deviations in the experimentally measured value of the root-mean-square roughness of a sample's surface. This point is also illustrated in Sec. V A of the present paper, where commercial AFM probes are imaged and discussed. Another common and often erroneous assumption is that the tip radius (whether known or unknown) does not change over the course of scanning. However, even at zero applied load, typical AFM probes of silicon and silicon nitride have been demonstrated to undergo significant shape change. ${ }^{16}$ Therefore, it can be critical for the tip shape to be accurately characterized at the beginning and end of an experiment. Direct imaging using electron microscopy (EM) provides a powerful tool for doing this. While algorithms exist for calculating the tip shape from measured SPM data (such as imaging of high-profile or undercut features, ${ }^{17,18}$ or using "blind tip reconstruction" computer routines, ${ }^{19,20}$ ) these are most powerful when combined with direct imaging, as discussed in Refs. 21 and 22. In the present work, a fixture has been designed for accurate, efficient, and facile imaging of AFM tips using electron microscopy.

\section{PREVIOUS WORK IMAGING NANOSCALE PROBES USING ELECTRON MICROSCOPY, AND DISCUSSION OF OPPORTUNITIES FOR IMPROVEMENT}

Electron microscopy represents a direct, straightforward, high-resolution method for imaging AFM probes. Many authors have fruitfully used electron microscopy to image scanning probe tips, as discussed in the following paragraph. A schematic showing the configuration and some key considerations is shown in Fig. 1. In a transmission electron microscope (TEM) experiment, the transmission geometry allows for the observation and measurement of a twodimensional profile of the outer contour of the tip. Tilting of the TEM goniometer can provide some additional information about the third dimension. Among other factors, contrast in TEM depends on the thickness through the imaging direction of the material; however, there are also contrast variations due to differences in composition (atomic number) and crystal structure. This often allows one to differentiate between a thin coating or an oxide layer and the rest of the tip (as shown in Fig. 1(b)). An additional significant benefit of TEM and scanning TEM (STEM) imaging is that information about crystal structure, composition, and chemical bonding can be obtained using analytical methods such as quantitative electron diffraction, energy dispersive x-ray spectroscopy (EDS), and electron energy loss spectroscopy (EELS). ${ }^{23}$

Many investigations have involved imaging of AFM probes in the TEM, most using custom-built fixtures. This work includes several examples from the study of wear of AFM probe tips in which a probe was imaged in an SEM or TEM before and after a wear experiment performed in the AFM. ${ }^{16,24-28}$ By measuring the side profile of the AFM probe tip, the authors measured the change in volume to describe wear or contamination of the tip. There are other investigations in the scanning probe literature (such as Refs. 21 and 29) where SEM/TEM was used to measure tip shape, so that robust values could be used in subsequent calculations. Many of these and related investigations, as well as our own past experiments, use custom-built fixtures. In addition to the time and effort required to design and build such fixtures, they can suffer three distinct disadvantages that can add significant uncertainty to measured results: (1) irreproducibility of alignment between subsequent mountings; (2) drift during imaging and contamination due to the use (in some) of carbon tape as a fixation method; and (3) wasted time and materials due to low throughput and tip breakage. Each is discussed, in turn, in the following paragraphs.

The first common disadvantage is that most of the fixturing methods reported previously in the literature did not include specific features to ensure reproducibility of the orientation of the tip being imaged. Variability in the rotational positioning of the tip from measurement to measurement, for example, could lead to uncertainty in the determination of the tip shape and the location of the point where the sample makes contact with the tip. Furthermore, variability in the orientation of the mounting hampers the ability to compare probes before and after testing in another apparatus, which is needed to determine whether there has been a change in the tip shape. Based on the present authors' prior experience with earlier fixturing, the position of the tip can deviate by more than one millimeter in all three spatial axes (in some cases locating the tip outside of the accessible range of the TEM goniometer), and the orientation of the conical tip can be rotated by tens of degrees about all three axes of rotation. Significant user skill is required to mount the probes in a similar orientation before and after testing; it is typically impossible to achieve identical orientation. Examples of this are shown in Fig. 2 where before/after images indicate changes in shape and in tip appearance.

Second, in cases where the probe needs to be used after TEM imaging (and thus cannot be permanently mounted such as using epoxy), carbon tape ${ }^{30}$ or adhesive glue ${ }^{31}$ is commonly used to secure the AFM probe. The use of carbon tape and some adhesive glues has been observed by the present authors to cause drift of the probes in the TEM and, in some cases, to introduce additional contamination into the TEM chamber. Carbon tape is a double-sided conductive adhesive tape, which is a widely used and highly versatile mounting tool for electron microscopy applications. However, the adhesive tape exhibits a time-dependent viscoelastic response that can cause positional drift with time and exhibits thermal expansion. Thus, if the tape is strained during mounting, or if the temperature inside the TEM chamber is different than that of the room, the tape will exhibit shape fluctuations and the position of the probe will translate with time. These factors hinder the otherwise highly precise atomic-scale measurements of the tip geometry possible in a TEM. Additionally, uncovered portions of the adhesive tape can collect carbonaceous contamination and other ambient room debris prior to insertion into the TEM chamber. Contamination can then be introduced into the TEM chamber when the electron beam irradiates the carbon tape (as it commonly does during initial low-magnification imaging of the probes), leading to outgassing and decomposition of the adhesive material, forming hydrocarbon contamination inside 

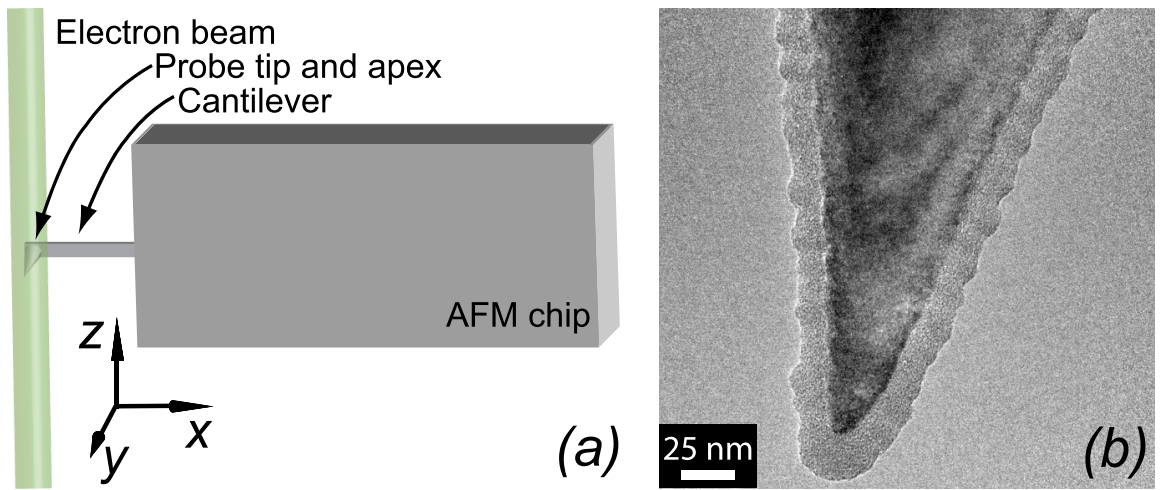

FIG. 1. An AFM probe is shown schematically (a) under a transmission electron microscope, with the relevant axes and features labeled. A canonical TEM image (b) demonstrates the contrast between the thin coating of diamond-like carbon and the single-crystal silicon probe beneath.

the chamber that redeposits on the tip and increases sample chamber pump-down time.

The third primary disadvantage of previous ad hoc mounting schemes is inefficient use of time and materials due to low throughput and high risk of tip breakage. Often, only one probe can be inserted into the TEM at a time in such fixtures. Thus, probes must be imaged sequentially with removal/re-insertion of the specimen transfer arm between each, which includes several minutes of pump-down. Additionally, ad hoc fixtures do not include a calibration grid or standard sample, so any beam alignment or calibration process requires additional sample exchanges (i.e., user and instrument time). Further, many ad hoc fixtures require precise (and often challenging) tip placement on carbon tape or glues, which can lead to increased risk of tip breakage.

\section{INSTRUMENTATION AND ADVANTAGES OF THE NOVEL FIXTURE}

To address the limitations of typical ad hoc TEM mounting schemes for AFM, we designed, fabricated, and extensively tested an advanced fixture for imaging scanning probes. The fixture was specifically designed for use in JEOL 2010, JEOL 2010F, and JEOL 2100 TEM systems, but the design is adaptable to other TEMs provided sufficient pole piece separation exists (approximately $2 \mathrm{~mm}$ is required in
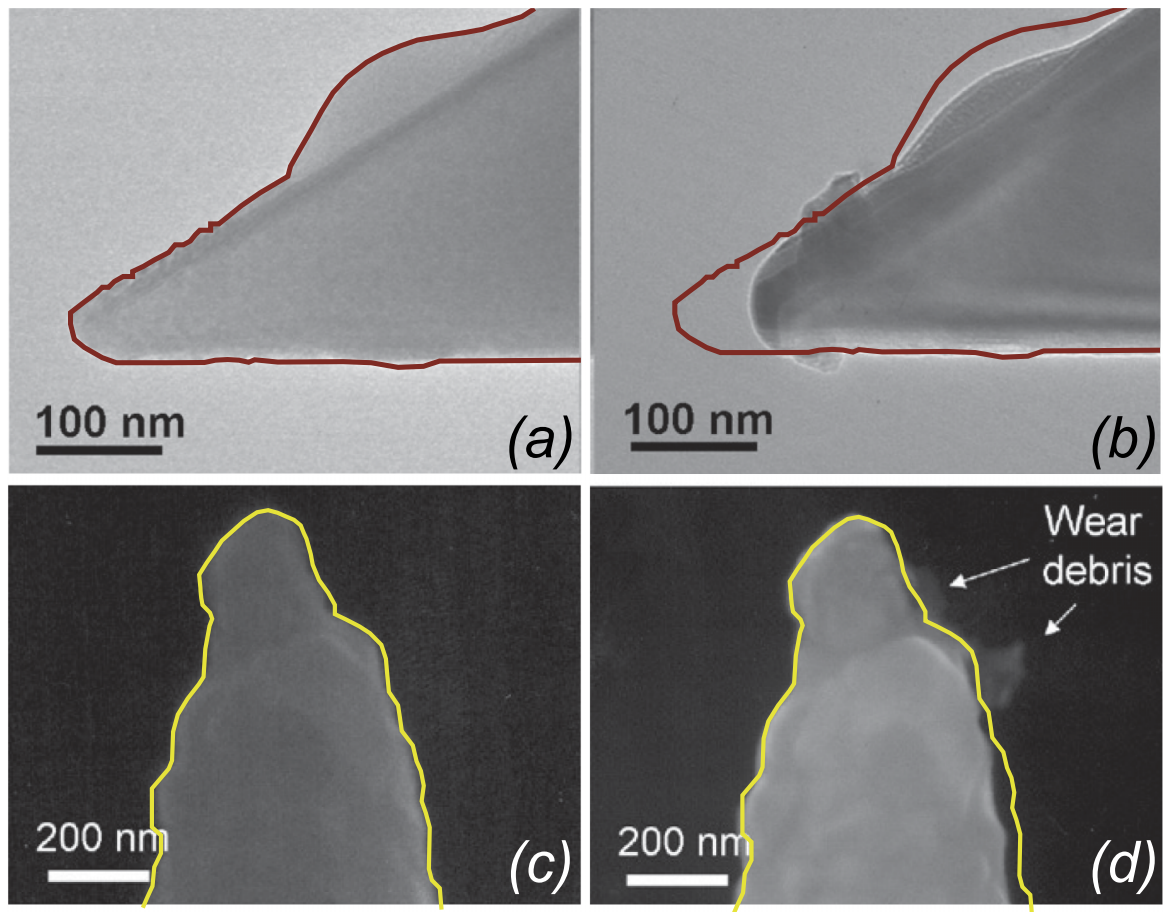

FIG. 2. Images taken from prior reports demonstrate both the power of EM imaging of AFM probes, but also the need for a more reliable fixture. For instance, a tip was imaged in the TEM before (a) and after (b) a sliding experiment. While there is clear evidence of shape change due to wear, there are also changes in the tip shape and appearance far from the point of contact; these are most likely caused by a rotation of the tip about its central axis upon re-mounting in the TEM. A second experiment shows SEM images before (c) and after (d) sliding and demonstrates that the base of the probe appears thinner after testing, likely because the tip is being viewed along a different orientation. Plates (a) and (b) reproduced with permission from Liu et al., ACS Nano 4 (7), 3763 (2010). Copyright 2010 American Chemical Society. Plates (c) and (d) reproduced with permission from Chung and Kim, Ultramicroscopy 108, 1 (2007). Copyright 2007 Elsevier. ${ }^{26}$ The red and yellow contours were added by the present authors to guide the reader's eye. 
order to fit the width of the AFM chip between the pole pieces). The design can be readily adapted for use in TEMs of a different model and manufacturer. The fixture enables highly repeatable positional and rotational alignment of probes and eliminates the need for any carbon tape or adhesive. It also increases imaging throughput-accommodating three probes simultaneously, together with a calibration sampleand reduces probe breakage. The fixture can be fabricated using common machining methods and tooling-requiring only a mill and readily obtained end mills. Figure 3 shows the main components of the scanning probe TEM fixture system, which consists of the fixture itself (referred to here as the multi-probe fixture) that simultaneously secures three typical atomic force microscope probe chips, and a mounting base used to stabilize the fixture during insertion and extraction of the probes. Sections III A and III B describe the primary considerations for design, fabrication, and use. Full technical drawings of the multi-probe fixture and base are included in the supplementary material. ${ }^{32}$

\section{A. Design considerations}

The multi-probe fixture mates to a standard JEOL TEM specimen transfer arm and securely positions three AFM probes and a standard 3-mm TEM grid for sequential imaging in the TEM without sample exchange. The probes are inserted into the fixture slots so that the plane containing the tip axis, the long axis of the cantilever, and the chip length is normal

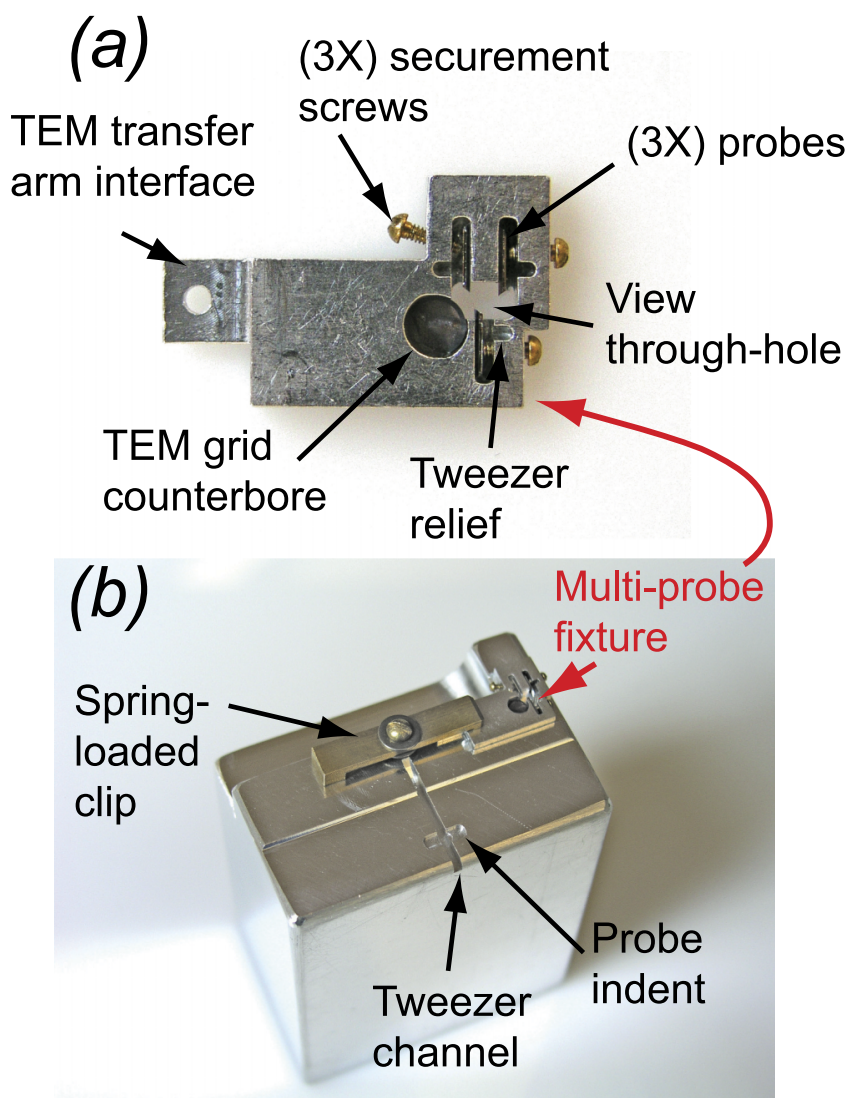

FIG. 3. An optical image is shown of the TEM multi-probe fixture (a), with salient features indicated. The mounting base (b) is a support mount which facilitates insertion and removal of AFM probes from the multi-probe fixture. to the TEM electron beam, with the cantilever overhanging a rectangular viewing window (through-hole). As shown in Fig. 4, this viewing window provides an unobstructed path to observe the side profile of the probe tip via the electron beam.

The lateral and vertical dimensions of the multi-probe fixture were selected to prevent interference between the fixture and the pole piece or the objective aperture of the TEM. Note that the TEMs used contain JOEL's analytical objective lens pole piece rather than their ultra-high resolution pole piece, which has a reduced gap. Details of the pole gap for any microscope can be obtained from the manufacturer. In general, contact between any of these elements could damage the probe fixture, transfer rod, or the TEM lenses. The dimensions were consequently tightly designed based on both the JEOL 2010/2100 specifications and the expected vertical travel needed to bring probe tips and the reference grid into focus. Furthermore, the exterior dimensions of the fixture were designed to fit within the maximum dimensions of the commercially available JEOL mount for TEM grids. The vertical depth of the probe fixture channels was designed so that the probe tips (typically positioned at the midpoint of the carrier chip) were aligned to the vertical position of the counterbore for the reference grid (a holey carbon sample) and with the nulled (zero) position of the probe fixture upon insertion. Accurate specification of the vertical positions ensures that little vertical adjustment is necessary from the zeroed position to focus on the probe tip. Finally, the multi-probe fixture is constructed using non-magnetic, nonferrous 6061 aluminum to minimize electromagnetic effects on the electron beam. Materials were also chosen to minimize outgassing or any other effects that would degrade the vacuum inside the TEM.

The probe slots were designed to securely position a wide range of commercial and custom AFM probes. Typical AFM probe carrier chips have a length, width, and depth of $3.4 \mathrm{~mm}, 1.6 \mathrm{~mm}$, and $0.315 \mathrm{~mm}$, respectively. While the slot dimensions were optimized for this typical probe geometry, larger and smaller custom sizes can be accommodated up to the length, width, and height of the slot itself due to the use of set screws. During loading, the AFM probes are gripped with tweezers and inserted into the slots. Each probe slot features a tweezer relief such that the user can open his/her tweezers after the AFM probe has been properly positioned in the slot. (Note that the tweezer relief only appears on the same side as the set screw and not on the opposite side; in previous design iterations, a tweezer relief opposite the set screw created conditions similar to a three-point bend test and resulted in increased probe breakage.) The addition of chamfers on the top edges of the slots could further ease probe insertion; however for fabrication simplicity, this is not included in the present design. During imaging the AFM probes are secured in the slots, with their cantilevers and tips extending over the through-hole for access from the electron beam. The slots were designed with lower shelves to ensure that probes will not fall out into the TEM column and to provide repeatable $\mathrm{z}$-axis positioning. Further, the lower shelf and the wall of the slot opposite the set screw provide orientation surfaces for repeatable mounting. 


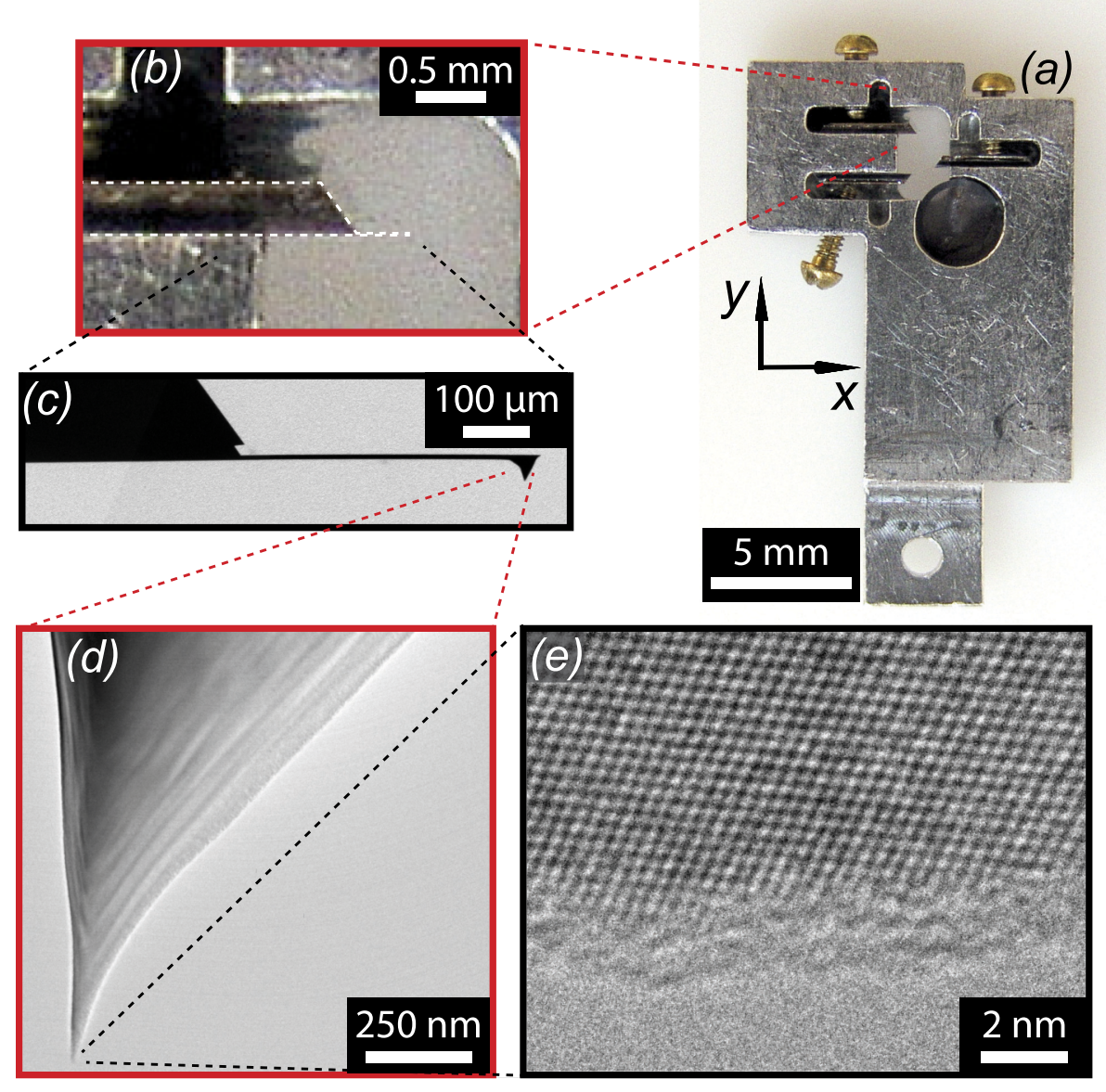

FIG. 4. Progressive zoom top-view images of the multi-probe fixture normal to the $x$-y plane (indicated in Fig. 1). In (a) the full part can be seen, with three carrier chips loaded and a reference grid. In (b) the carrier chip and cantilever are outlined to guide the eye. Low-magnification TEM images (c) enable visualization of the cantilever and its orientation. Intermediate-magnification TEM images (d) indicate the overall shape and aspect ratio of the tip. High-resolution images (e) can be used to ascertain the detailed shape of the apex; in many cases the atomic lattice can be resolved.

Brass set screws (size \#000, 120 threads per in.) are used to secure the probe in the probe slot. The probe cantilever is typically aligned opposite these screws to avoid potential cantilever breakage or contamination during tightening. Screws are tightened using a flathead jeweler screwdriver. To minimize the risk of probe breakage, the ends of the screws were rounded using 400 grit sandpaper to reduce stress concentrations where screw-chip contact occurs.

The multi-probe fixture includes a circular boss that accommodates a standard 3-mm-diameter TEM grid. This is typically fitted with a calibration standard, such as a "holey carbon" film for beam alignment and astigmatism correction, or an evaporated metal diffraction standard. These enable "on-the-fly" correction of imaging parameters such as stigmators and calibration of images without the need for sample removal/reinsertion or changing imaging conditions. The TEM grid is secured in the boss by applying a small amount of colloidal silver paste to the unused edge of the grid (far from the imaging through-hole).

Additionally, a mounting base (also known as a third hand) fixture was designed to aid in the mounting of AFM probes in the multi-probe fixture. The mounting base provides a sturdy, raised platform on which to work, and securely holds the multi-probe fixture to prevent moving and rotation during loading/unloading of chips. Further, the mounting base is designed with a transfer platform which enables the user to change tweezer grips (as described in Sec. III B).

The fixturing has also been designed for the simplest possible fabrication. The required tooling includes only end mills and twist drills, with just 3 relevant $\mathrm{z}$ heights. Further, all features are Cartesian (not arbitrary), thus only a manual mill with $\mathrm{x}, \mathrm{y}$, and $\mathrm{z}$ indicators is required. This obviates the need for computer numerical control.

\section{B. Basic probe insertion protocol}

Typical usage of the multi-probe fixture includes securing the fixture to the mounting base followed by inserting up to three AFM probes. Specifically, AFM probes are inserted along their long edge, such that the axes of the cantilever and the probe tip are normal to the TEM viewing screen. In this way, the probe tip radius may be imaged as shown in Fig. 4. Probes are loaded into each slot with the cantilever hanging over the through-hole window; this window provides an unobstructed path for the TEM electron beam.

The probe slot widths are approximately 3 times the thickness of a typical scanning probe carrier chip thickness, which provides room for tweezer points during loading and unloading. Tweezer reliefs have also been included on 
one side of each slot to further accommodate the tweezer points.

Upon final insertion, the AFM probe must be rotated by $90^{\circ}$ (about its longest axis) from the orientation it has in its commercial packaging. To facilitate the transition of orientation, a transfer platform was designed on the mounting base. This way, the user can pick the probe up from the commercial packaging and set it down (in the same orientation) on the transfer platform. Then, taking advantage of the underlying slot, the user can easily pick up the probe with their tweezer grip rotated by $90^{\circ}$ in preparation for final insertion.

The probes are secured in their slots using set screws, which are tightened with a jeweler's screwdriver. The screws must be sufficiently tight to ensure secure clamping while avoiding probe chip breakage. The appropriate amount of torque is determined by practicing on unneeded probes. We have found that, once determined, this torque is consistent for a wide range of probes. Once all probes are mounted, it is recommended to turn the multi-probe fixture upside down to ensure that the probes are secure. In the event of probe breakage upon overtightening, it is recommended to clean out debris using a pressurized air dust removal canister or a compressed gas gun. Remaining shards of broken probes can act as stress concentrators, increasing the risk of future fractured probes.

Once the probes are securely mounted, the multi-probe fixture can be inserted into the TEM for imaging.

\section{PRACTICAL CONSIDERATIONS FOR ACCURATE IMAGING OF AFM TIPS}

The present fixture has sufficient stability to routinely permit lattice-resolution imaging of AFM tips. The present authors have imaged probes up to more than one million times magnification, even for flexible contact-mode cantilevers with spring constants of approximately $0.1 \mathrm{~N} / \mathrm{m}$. For ultra-soft cantilevers $(<0.05 \mathrm{~N} / \mathrm{m})$, thermal vibration at the cantilever's resonance frequency begins to blur the images and can preclude lattice resolution; however, overall tip size/shape is still easily attainable. As discussed, the present fixture enables a significant improvement over other fixtures in reproducibility of probe mounting - this enables reliability of shape measurement and increased fidelity of comparisons before and after probe remounting. Below are three primary considerations to attain the highest quality probe imaging: orientation, charging, and contamination/degradation. Each is discussed in the context of both advantages of the present fixture, and also "best practices" for repeatable imaging of AFM probes.

The orientation of the AFM probe in the TEM is critical for reliable, quantitative analysis of the nanoscale tip. Even for a single measurement, the tip can appear to be foreshortened if the probe is rotated about the long axis of the cantilever. For comparison measurements (e.g., comparing TEM images of the same probe taken before and after a wear test that was performed in an AFM), reproducible orientation is imperative for reliable detection of qualitative changes (e.g., structural defects) or quantitative characterization (e.g., measurement of probe radius or material removal). To maximize alignment reproducibility, the probe should be mounted in the same slot (of the three) and with the tip pointing in the same direction.

With regard to rotation of the probes upon insertion, the lower shelves and side walls of the probe slots prevent tilting about two axes; rotation about the third axis-along the long axis of the probe tip-is controlled using alpha-tilt of the TEM goniometer. For AFM probes made from single-crystal materials, TEM diffraction pattern imaging can be used to identify a specific high-symmetry orientation and all imaging can be performed at this specific orientation. For silicon probes mounted in the current fixture, the [110] zone axis can be readily found (as shown in Fig. 5(a)) and images can be taken at that orientation. Small rotations away from a zone axis will cause a characteristic ring of bright spots (Fig. 5(b)), which will decrease in radius as the probe is tilted towards the zone axis. Therefore, after the probe is removed, used in an AFM, and re-mounted in the TEM, goniometer tilt can be used to return to the identical orientation-as confirmed by the diffraction image. For non-crystalline or polycrystalline

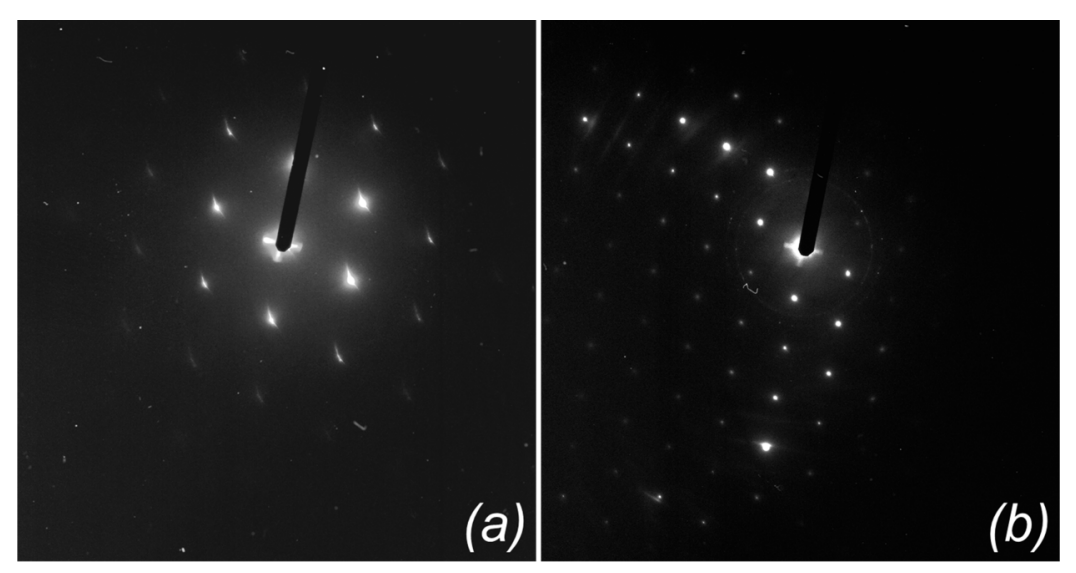

FIG. 5. Selected area diffraction can be used to demarcate specific orientations of the probe tip. When the orientation of the single-crystal silicon probe is such that the electron beam lies along (or very near to) a high symmetry axis, then a recognizable spot pattern is observed (a). When the crystal is tilted away from this high-symmetry axis, then a modified spot pattern is observed (b). In the latter case, tilt about the long axis of the specimen transfer arm can typically be used to approach the high symmetry orientation. 
samples, a similar procedure can be used, but with apparent tip shape (observed by bright field TEM imaging) as the metric for alignment. The latter method is particularly useful in cases where imperfections or small pieces of contamination on the shank of the probe serve as fiducial markers; however it is invariably less reliable than using the diffraction pattern of single crystals.

Another consideration in TEM imaging of AFM probes is charging. Most commercial AFM probes are designed to be conductive such that static charge build-up does not affect approach or contact with surfaces-thus, charging in the TEM is not typically a problem. Nevertheless, certain specialty cantilevers (such as undoped diamond probes, or conductive cantilevers on insulating carrier chips) can have low electrical conductivity and may charge in the TEM. This causes significant motion and drift of the tip, particularly for softer cantilevers, and precludes high-resolution imaging. This can often be resolved by sputter-coating a layer of metal (typically $\sim 100 \mathrm{~nm}$ of $\mathrm{Al}$ or $\mathrm{Pt}$ ) on the backside of the cantilever. In some cases, the AFM chip is built on a carrier chip composed of Pyrex or another insulating material. For these probes, it is occasionally necessary to apply a conductive coating (carbon paint, or conductive epoxy) to the side surface of the carrier chip that is closest to the electron beam. This prevents static charge build-up in the carrier chip, which can sometimes distort the electron beam. In summary, most probes need no preparation whatsoever to be imaged in the TEM; and in a very few cases where charging is problematic, it can typically be readily resolved.
A final consideration for imaging probes (or any other sample) in the TEM is contamination or damage. Standard TEM practices should be employed for the imaging of AFM probes-these include: allowing the column to pump down sufficiently before imaging; using the minimum possible electron current to achieve the necessary magnification; minimizing time of exposure to the electron beam; reducing accelerating voltage of the TEM for beam-sensitive materials; testing the effect of the imaging on an alternate tip before imaging an important one; and minimizing the amount of carbon tape or other carbon-based adhesives/coatings that can degrade to contaminants under the electron beam. The present fixture improves many of these considerations-for instance, the pre-alignment of the probe reduces imaging time used for orientation and the lack of carbon tape or adhesives reduces the amount of carbonaceous material in the chamber.

With appropriate care, the present authors have been able to image a large number of probes with lattice resolution without evidence of contamination build-up, or extensive knock-on damage. It has been observed, for instance, that: metal-coated probes are extremely robust to beam exposure; standard silicon probes are relatively unaffected, though the surface oxide can deform spontaneously under very high electron beam current; diamond-like carbon coatings can be readily imaged, but some compositions suffer degradation and material removal at high magnification, or the formation of adlayers. For all materials, a pre-characterization study should be performed to determine the beam-sensitivity and develop strategies to mitigate.

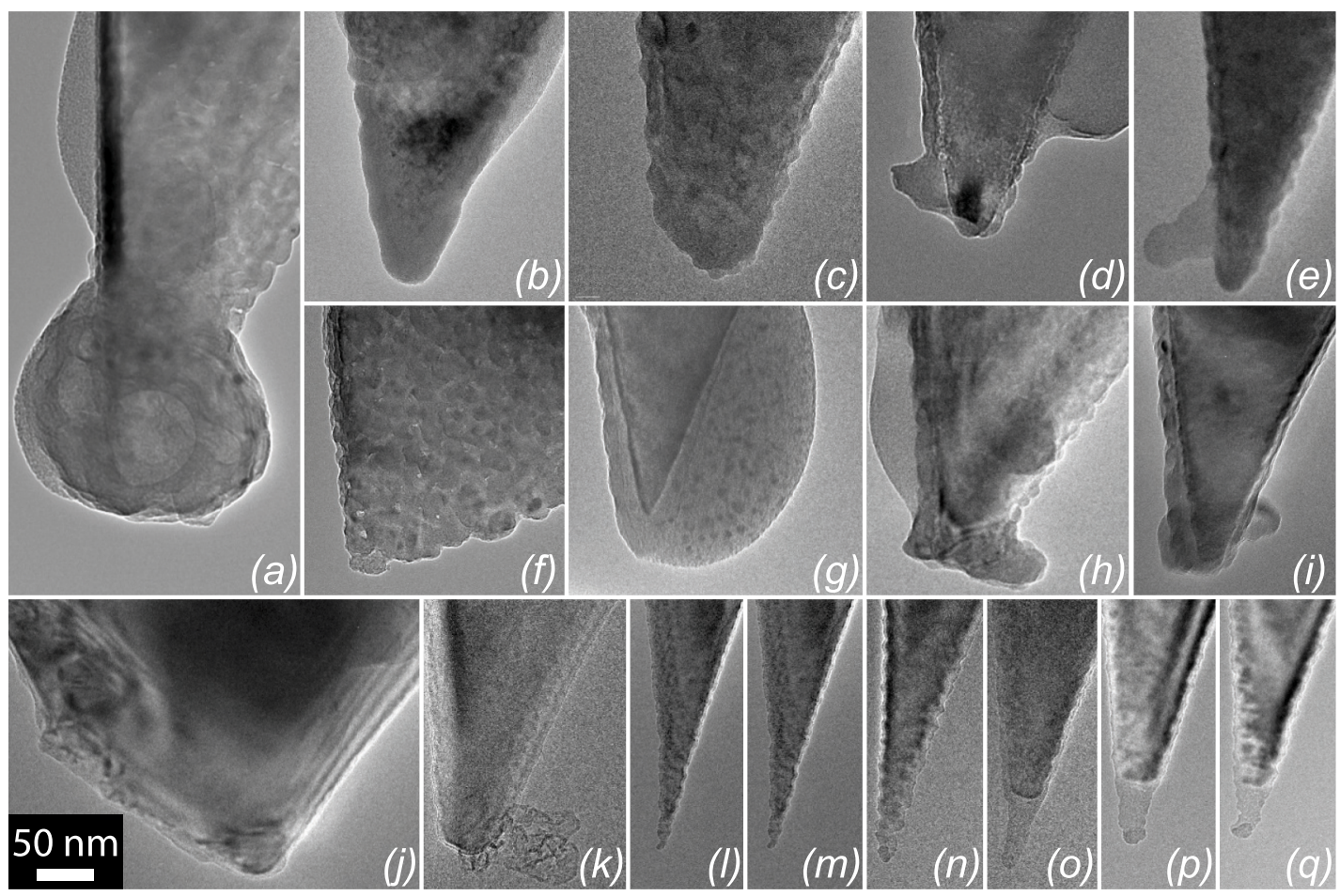

FIG. 6. Examples of 17 commercial silicon cantilevers, all at a common magnification. The top nine probes are all of a single model from a single manufacturer, while the bottom eight are all of a single model from a different manufacturer; both manufacturers' products are commonly used in scanning probe microscopy. All probes are shown "out of the box," after only minimal handling by an experienced user. These probes demonstrate a wide range of tip conditions, tip radii, and tip contamination. While it is possible that damage or contamination occurred during handling, the handling was roughly equivalent to the amount required for mounting an AFM probe for use in an AFM. Therefore, these images (and the wide variety of tip shapes/sizes/conditions) should be considered representative of the state of probes used during standard scanning probe microscopy measurements. Note that the scale bar in (j) applies to all images shown. 


\section{EXAMPLES OF USE AND CHARACTERIZATION OF PROBE TIPS}

\section{A. Tip profiles of as-received commercial cantilevers}

A survey was conducted of common commercial cantilevers in their out-of-the-box state. Nine probes from one manufacturer and eight probes from a different manufacturer were examined in the TEM at a common magnification, as shown in Fig. 6. This survey demonstrates the extreme variation from probe to probe-even when the probes are of identical type, or taken from the same package. In fact, the tips shown in Figs. 6(d) and 6(e) are on the same AFM probe (that particular variety has three cantilevers per chip). This means that they underwent the same processing steps under identical conditions-yet there are obvious differences in shape, aspect ratio, and contamination.

This survey demonstrates the importance of TEM imaging of AFM probes and that the manufacturers' estimates of probe tips may be significantly in error. Further, beyond the size of the probe tip, it is shown that the tip material can vary widely as well. All of these probes are nominally made from single-crystal silicon. Yet while some probes demonstrate only a thin native oxide over the end of the probe, the TEM images show that others have a large lighter-colored region at the end that-upon closer inspection-is shown to be amorphous silicon oxide. This has ramifications for quantitative studies of mechanical properties, conductivity, friction, and wear resistance. Further, several probes show contamination, which appears to be carbonaceous, that is covering the end of the probe. This can further complicate quantitative SPM studies. A final consideration that is made clear from Fig. 6 is that while one of the manufacturer's tips has consistently smaller end radii than the other, they also have extremely high aspect ratio, which increases the risk of tip fracture. Because tip fracture can lead to extremely blunt, flat-ended probes (somewhat similar to the probe shown in Fig. 6(j)), then the best choice for use will depend on desired scanning conditions and skill of the user.

\section{B. Examples of repeatable probe orientation for accurate determination of wear}

The ability to achieve repeatable positioning of the probes is demonstrated by wear experiments performed in an AFM, with before and after images taken in the TEM. A contact-mode AFM probe (of the same model as those shown in Figs. 6(j)-6(q) was mounted on the present fixture and imaged (Fig. 7(a)) using the best practices outlined in Sec. IV. It was then removed from the TEM, taken to an AFM (Multimode, Veeco, now part of Bruker), and scanned for $20 \mathrm{~mm}$ at a constant applied load of $3 \mathrm{nN}$ on a thin film of tetrahedral amorphous carbon $(\mathrm{t}-\mathrm{aC})$ deposited on a silicon wafer. It was then re-mounted on the present fixture and re-imaged (Fig. 7(b)) in the TEM. The diffraction orientation technique enabled precise re-positioning of the probe, such that the outline of the unworn portion of the probe can be exactly matched in the before and after condition. Since orientational artifacts can thus be ruled out, the change in tip appearance can be confidently attributed to material removal during testing. A second test was run (Figs. 7(c) and 7(d)) using a probe of the same model as those shown in Figs. 6(a)-6(i) — but under different sliding conditions (3.42 m of sliding on an uncoated silicon wafer at a constant applied load of $3 \mathrm{nN}$ ). Once again, changes in appearance at the apex of the probe can be attributed to damage during testing rather than imaging artifacts and a quantitative analysis of tip wear can be performed.

Finally, an ultrananocrystalline diamond probe with a triangular profile was used, not for a wear study, but for a "tilt

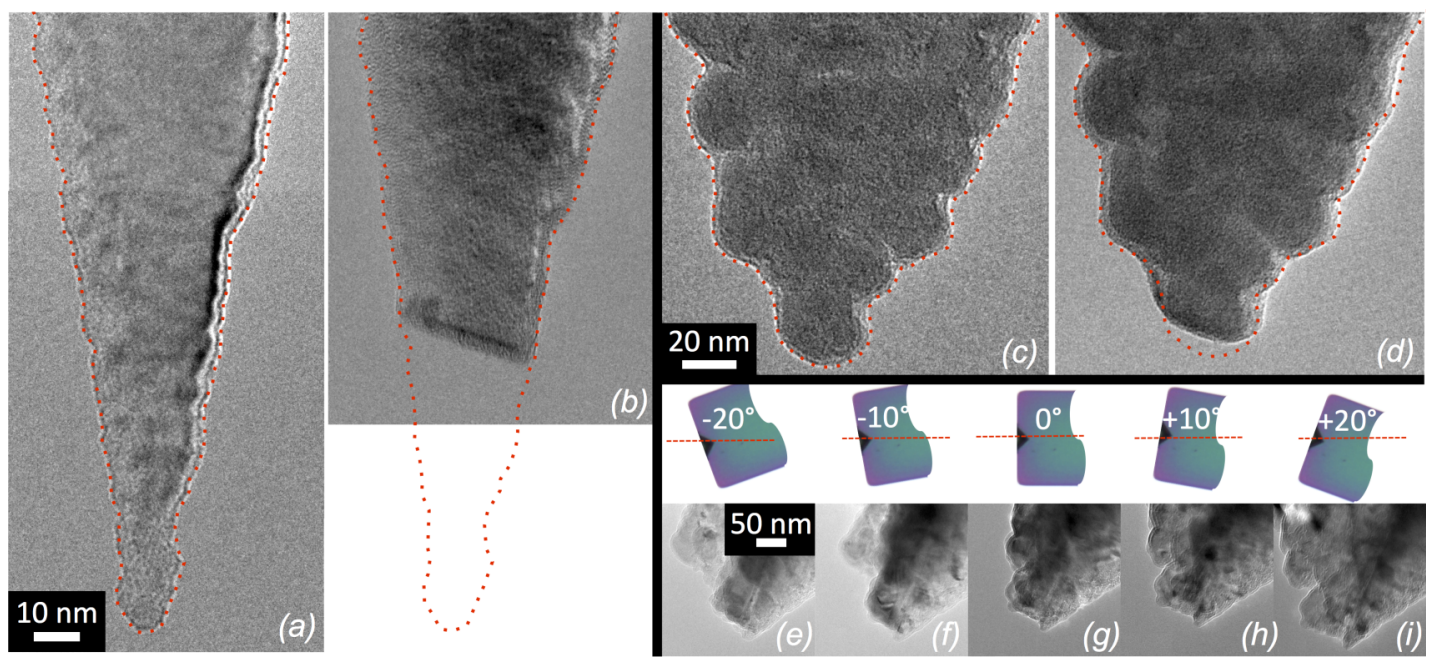

FIG. 7. Two wear studies and a tilt study demonstrate the ability of, and importance of, reproducible tip orientation. Two different probes were imaged in the TEM in their as-received condition ((a) and (c)) using the present fixture and best-practices for imaging. They were removed for wear testing in an AFM (under different scanning conditions), and then re-mounted and re-imaged in the TEM after testing ((b) and (d)). The red dotted outlines superimposed on the before and after images show a near-perfect match and demonstrate that changes in the tip apex are meaningful and are not due to TEM artifacts. In contrast, ((e)-(i)) demonstrate the potential for non-meaningful changes in tip appearance in the TEM. An ultrananocrystalline diamond probe is imaged at five different orientations (shown schematically above each image). There is significant change in tip appearance due to misorientation, which has the potential to increase uncertainty in tests that do not precisely control for tip orientation. 
study" to determine the change in appearance that can result from tilting alone. As shown in Figs. 7(e)-7(i), the same probe is imaged in five different tilt configurations (as indicated in the figures), and significant changes in apparent tip shape are visible. In the present case-where tilt was well controlled and no changes in the tip occurred-it is easy to attribute these changes to imaging conditions. However, this demonstrates that if a probe were removed for testing and unintentionally remounted at a different angle, then such changes could be mistakenly attributed to wear, contamination, or other meaningful tip change.

\section{Repeatable probe orientation for the observation of tribopolymer growth and material displacement on metal-coated probes}

The repeatable positioning afforded by the multi-probe fixture described in this report enables reliable observation of nanoscale tip information even when zone axis information is unattainable. SPM studies often require coating commercial Si probes with thin layers of polycrystalline materials ${ }^{33-36}$ or the entire probe may be composed entirely of such materials. ${ }^{37,38}$ Consequently, the diffraction orientation technique discussed in Section V B cannot be used to align the imaging plane between multiple mountings (e.g., before and after testing in an AFM). However, as mentioned above, the integrated datums in our multi-probe fixture facilitate repeatable probe orientation.

The study of nanoscale electrical contact degradation of metal-coated AFM probes demonstrates the benefit of repeatable probe positioning. In this experiment, a Ptcoated commercial Si AFM probe tip was brought into contact with a $\mathrm{Pt}$ counter-surface for $2 \times 10^{9}$ cycles to mimic the conductive interface of a nanoscale switch. ${ }^{33,39}$
The contact resistance of the Pt-Pt interface undesirably increased by an order of magnitude after cycling, which corroborates observations of contact fouling in macroand microscale metal contacts. ${ }^{40,41}$ These previous studies attributed resistance increases to tribomechanical growth of insulating films (termed "tribopolymers"), which have been previously identified and characterized by optical or EM imaging of planar interfaces ${ }^{41-43}$ or chemical analysis. ${ }^{41}$ In the experiment here, the small dimensions of the AFM probe tip and the ability to reproducibly orient the imaging plane of the probe tip allow direct EM observation of displaced material and contaminant growth as shown in Fig. 8 .

The polycrystalline $\mathrm{Pt}$ coating of the probe in Fig. 8 obscures the underlying single crystal Si probe, and precludes the use of the diffraction alignment technique. Regardless, because of the mounting channel datums, z-axis variations were routinely observed to be within $1^{\circ}$ when re-imaging (removing and reinserting) probes. It is reasonably assumed that rotation around the $y$-axis, which affects the image plane, is equivalent to or less than $\mathrm{z}$-axis variations. Consequently, comparisons between micrographs of the probe from separate imaging sessions were always found to register with minimal distortion when overlaid. The precision of the fixture alignment, particularly with respect to rotations around the $\mathrm{x}$ - and $\mathrm{y}$-axes, confers confidence when observing adlayers - that is, accumulated material on the probe tip may be attributed to additional material instead of the appearance of additional facets of material resulting solely from rotation of the probe viewing plane. Repeatable positioning of probes like that shown here is aided by ensuring planarity, parallelism, and perpendicularity when manufacturing the multi-probe fixture and routinely cleaning the probe fixture channels/datums.

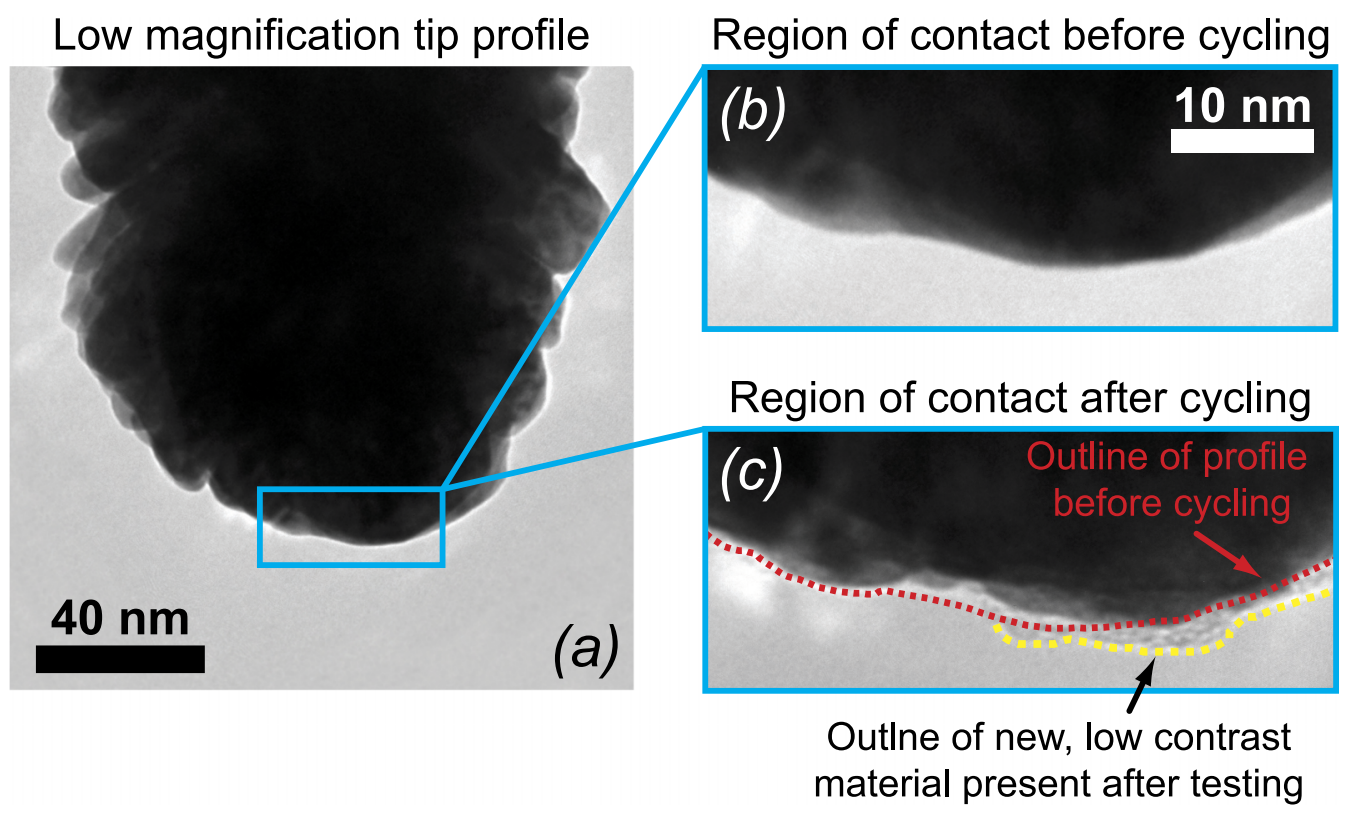

FIG. 8. A commercial Si AFM probe coated with $70 \mathrm{~nm}$ of Pt was brought into contact with a Pt surface $2 \times 10^{9}$ times and imaged before and after cycling using TEM. A low-magnification view (a) shows the faceted profile of the probe tip. Expanded views of the point of contact of the probe before (b) and after (c) cycling show material removal and buildup of additional, low-density (low-contrast) tribopolymer material. The ability to accurately align the probe tip along the $\mathrm{x}$ - and $\mathrm{y}$-axes ensures that the appearance of new material is not simply new facets of material revealed solely due to probe tilt. 


\section{CONCLUSIONS}

This paper presents a novel fixture and a description of the methodology and use for accurate imaging of probe tips for SPM using a TEM. It is argued that general practitioners of quantitative SPM techniques can benefit from TEM imaging of probes because many analyses rely on (or can be improved by) accurate knowledge of the geometry and composition of the probe tip. Further, a survey of out-of-the-box commercial probes from common manufacturers demonstrates a wide range of shape, size, and composition. Therefore, we present the design, fabrication, and use of a fixture to enable easy and precise positioning of the probe for use in the TEM. We present best practices for the TEM imaging to achieve reliable, non-destructive tip evaluation-which can even be used before and after other testing to detect tip changes or to prove the lack thereof. Finally, imaging for two ex situ wear tests is presented to demonstrate the reproducibility of tip imaging for accurate, quantitative comparisons.

\section{ACKNOWLEDGMENTS}

The authors gratefully acknowledge design and fabrication help from Mr. Peter Rockett and Dr. Doug Yates. We thank the Nanoscale Characterization Facility in the Singh Center for Nanotechnology, University of Pennsylvania, for use of facilities. R.W.C. and T.D.B.J. acknowledge support from National Science Foundation under award No. CMMI12-00019. R.W.C. acknowledges support from AFOSR under Contract No. FA2386-15-1-4109 AOARD.

${ }^{1}$ D. Grierson, E. Flater, and R. Carpick, J. Adhes. Sci. Technol. 19, 291 (2005).

${ }^{2}$ P. L. Piotrowski, R. J. Cannara, G. Gao, J. J. Urban, R. W. Carpick, and J. A. Harrison, J. Adhes. Sci. Technol. 24, 2471 (2010).

${ }^{3}$ T. D. B. Jacobs, J. A. Lefever, and R. W. Carpick, Tribol. Lett. 59, 1 (2015).

${ }^{4}$ T. D. B. Jacobs, J. A. Lefever, and R. W. Carpick, Adv. Mater. Interfaces 2 , 1400547 (2015).

${ }^{5}$ P. Egberts and R. Bennewitz, Nanotechnology 22, 425703 (2011).

${ }^{6}$ R. Bennewitz, K. Brörmann, P. Egberts, N. N. Gosvami, F. Hausen, and C. Held, Adv. Eng. Mater. 12, 362 (2010).

${ }^{7}$ M. Kopycinska-Müller, R. H. Geiss, and D. C. Hurley, Ultramicroscopy 106, 466 (2006).

${ }^{8}$ D. C. Hurley, M. Kopycinska-Müller, A. B. Kos, and R. H. Geiss, Meas. Sci. Technol. 16, 2167 (2005)

${ }^{9}$ R. W. Carpick, N. Agrait, D. F. Ogletree, and M. Salmeron, J. Vac. Sci. Technol., B: Microelectron. Nanometer Struct. 14, 1289 (1996).

${ }^{10}$ R. Carpick, N. Agrait, D. Ogletree, and M. Salmeron, Langmuir 12, 3334 (1996).
${ }^{11}$ D. S. Grierson, J. Liu, R. W. Carpick, and K. T. Turner, J. Mech. Phys. Solids 61, 597 (2013).

${ }^{12}$ H.-J. Butt, B. Cappella, and M. Kappl, Surf. Sci. Rep. 59, 1 (2005).

${ }^{13}$ T. D. B. Jacobs, K. E. Ryan, P. L. Keating, D. S. Grierson, J. A. Lefever, K. T. Turner, J. A. Harrison, and R. W. Carpick, Tribol. Lett. 50, 81 (2013).

${ }^{14}$ S. Kalinin and D. Bonnell, Phys. Rev. B 65, 125408 (2002).

${ }^{15}$ D. L. Sedin and K. L. Rowlen, Appl. Surf. Sci. 182, 40 (2001).

${ }^{16}$ J. Liu, J. K. Notbohm, R. W. Carpick, and K. T. Turner, ACS Nano 4, 3763 (2010).

${ }^{17}$ L. Montelius and J. O. Tegenfeldt, Appl. Phys. Lett. 62, 2628 (1993).

${ }^{18}$ J. Vesenka, R. Miller, and E. Henderson, Rev. Sci. Instrum. 65, 2249 (1994).

${ }^{19}$ J. S. Villarrubia, J. Res. Natl. Inst. Stand. Technol. 102, 425 (1997).

${ }^{20}$ E. E. Flater, G. E. Zacharakis-Jutz, B. G. Dumba, I. A. White, and C. A. Clifford, Ultramicroscopy 146, 130 (2014).

${ }^{21}$ J. A. DeRose and J. P. Revel, Microsc. Microanal. 3, 203 (1997).

${ }^{22}$ V. Vahdat, D. S. Grierson, K. T. Turner, and R. W. Carpick, ACS Nano 7, 3221 (2013).

${ }^{23}$ D. B. Williams and C. B. Carter, Transmission Electron Microscopy: A Textbook for Materials Science (Springer, 2009).

${ }^{24}$ B. Gotsmann and M. A. Lantz, Phys. Rev. Lett. 101, 125501 (2008).

${ }^{25} \mathrm{~K}$. Chung, Y. Lee, and D. Kim, Ultramicroscopy 102, 161 (2005).

${ }^{26} \mathrm{~K}$. Chung and D. Kim, Ultramicroscopy 108, 1 (2007).

${ }^{27}$ K.-H. Chung, Y.-H. Lee, H.-J. Kim, and D.-E. Kim, Tribol. Lett. 52, 315 (2013).

${ }^{28}$ V. Vahdat, K. E. Ryan, P. L. Keating, Y. Jiang, S. P. Adiga, J. D. Schall, K. T. Turner, J. A. Harrison, and R. W. Carpick, ACS Nano 8, 7027 (2014).

${ }^{29}$ C. Lee, X. Wei, J. W. Kysar, and J. Hone, Science 321, 385 (2008).

${ }^{30}$ H. Bhaskaran, B. Gotsmann, A. Sebastian, U. Drechsler, M. A. Lantz, M. Despont, P. Jaroenapibal, R. W. Carpick, Y. Chen, and K. Sridharan, Nat. Nanotechnol. 5, 181 (2010).

${ }^{31}$ G. Huttl, V. Klemm, R. Popp, and F. Simon, Surf. Interface Anal. 33, 50 (2002).

${ }^{32}$ See supplementary material at http://dx.doi.org/10.1063/1.4937810 for full technical drawings of the multi-probe fixture and the mounting base.

${ }^{33}$ F. Streller, G. E. Wabiszewski, and R. W. Carpick, IEEE Nanotechnol. Mag. 9, 18 (2015).

${ }^{34}$ H. Hosoi, M. Kimura, K. Hayakawa, K. Sueoka, and K. Mukasa, Appl. Phys. A 72, S23 (2014).

${ }^{35}$ H. Bhaskaran, A. Sebastian, and M. Despont, IEEE Trans. Nanotechnol. 8, 128 (2009).

${ }^{36}$ X. D. Cui, A. Primak, X. Zarate, J. Tomfohr, O. F. Sankey, A. L. Moore, T. A. Moore, D. Gust, G. Harris, and S. M. Lindsay, Science 294, 571 (2001).

${ }^{37}$ J. Liu, D. Grierson, N. Moldovan, J. Notbohm, S. Li, P. Jaroenapibal, S. O'Connor, A. Sumant, N. Neelakantan, and J. Carlisle, Small 6, 1140 (2010).

${ }^{38}$ E. Bussmann and C. C. Williams, Rev. Sci. Instrum. 75, 422 (2004).

${ }^{39}$ F. Streller, G. E. Wabiszewski, and R. W. Carpick, in Proceedings From the IEEE Conference on Nanotechnology (IEEE, Toronto, Canada, 2014), pp. 141-145.

${ }^{40}$ D. A. Czaplewski, C. D. Nordquist, C. W. Dyck, G. A. Patrizi, G. M. Kraus, and W. D. Cowan, J. Micromech. Microeng. 22, 105005 (2012).

${ }^{41}$ L. Chen, H. Lee, Z. J. Guo, N. E. McGruer, K. W. Gilbert, S. Mall, K. D. Leedy, and G. G. Adams, J. Appl. Phys. 102, 074910 (2007).

${ }^{42}$ H. W. Hermance and T. F. Egan, Bell Syst. Tech. J. 37, 739 (1958)

${ }^{43}$ V. Brand, M. S. Baker, and M. P. de Boer, Tribol. Lett. 51, 341 (2013). 
Supplementary materials for:

\section{Characterizing Nanoscale Scanning Probes Using Electron Microscopy: A Novel Fixture and a Practical Guide}

Tevis D. B. Jacobs, Graham E. Wabiszewski, Alexander J. Goodman, Robert W. Carpick

Prof. T. D. B. Jacobs

538-E Benedum Hall, 3700 O'Hara St, Pittsburgh, PA, 15208, USA

Email: tjacobs@pitt.edu

Dr. G. E. Wabiszewski

272 Towne Bldg, 220 S. $33^{\text {rd }}$ St., Philadelphia, PA, 19104, USA
A. J. Goodman
112 Towne Bldg, 220 S. $33^{\text {rd }}$ St., Philadelphia, PA, 19104, USA
Prof. R. W. Carpick
229 Towne Bldg, 220 S. $33^{\text {rd }}$ St., Philadelphia, PA, 19104, USA
Email: carpick@seas.upenn.edu
Keywords: TEM, fixture, AFM, probe, nanoscale, characterization
S1. Technical drawings for the probe holder fixture
Below are all of the drawings and specifications needed for a machinist to create the present probe holder fixture and the complementary mounting base. 


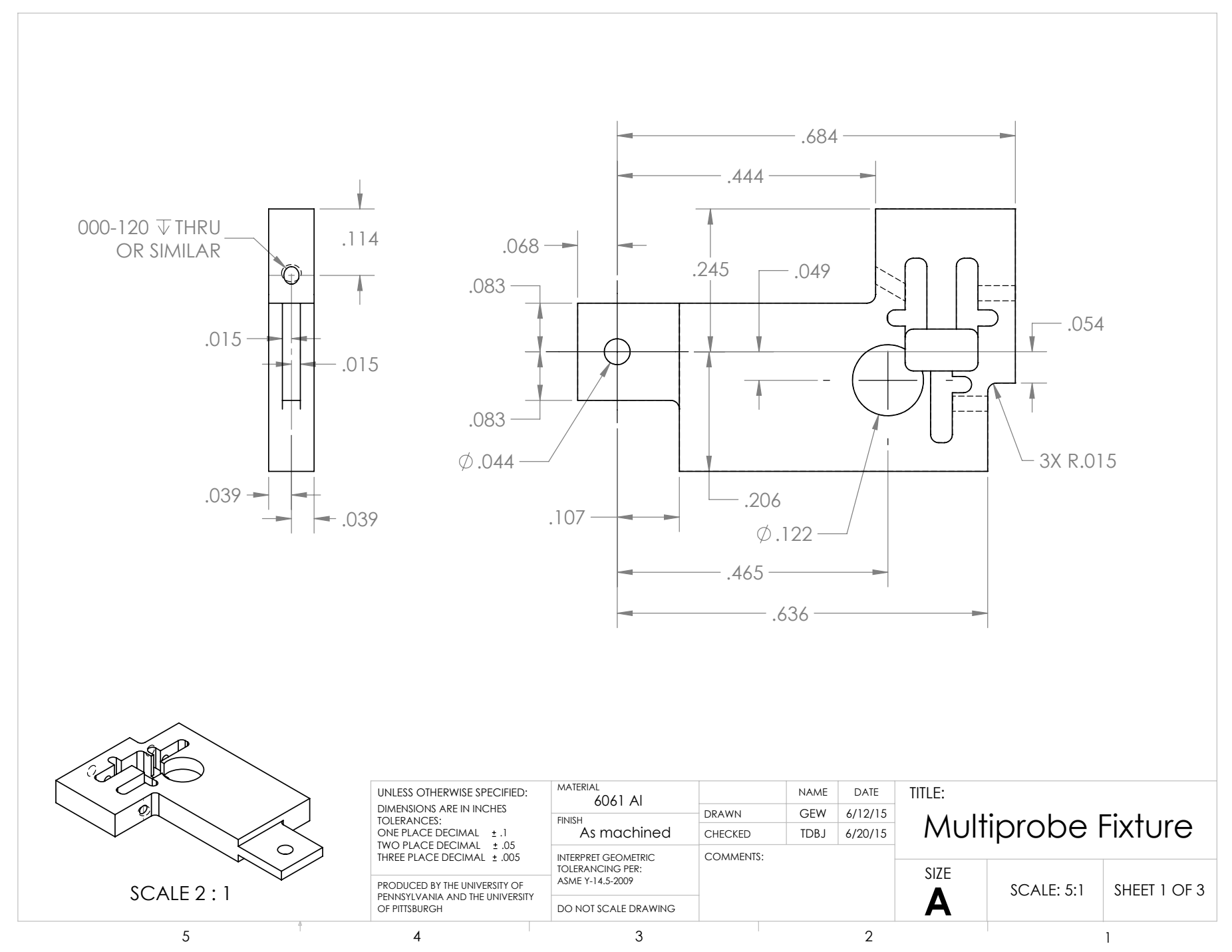




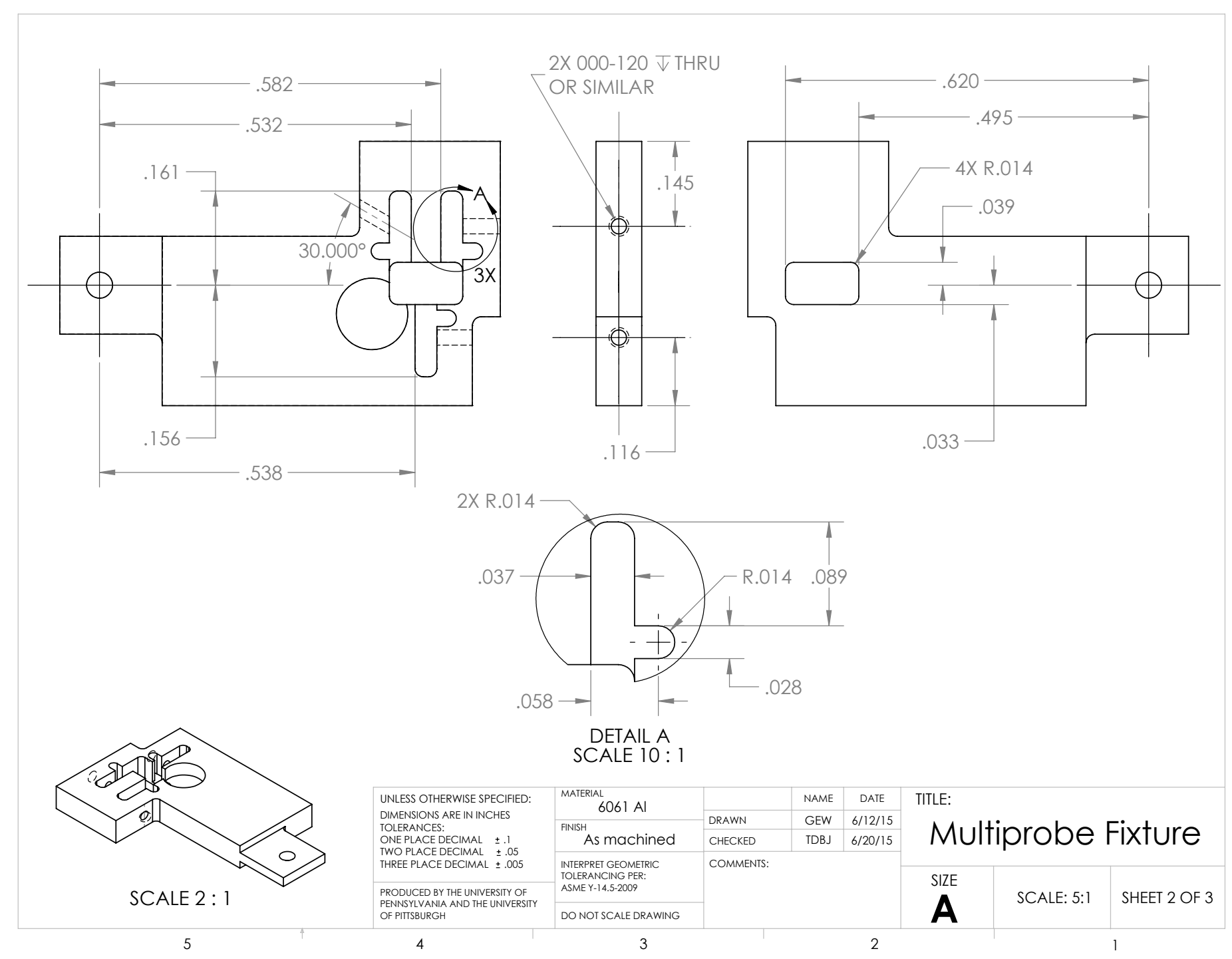




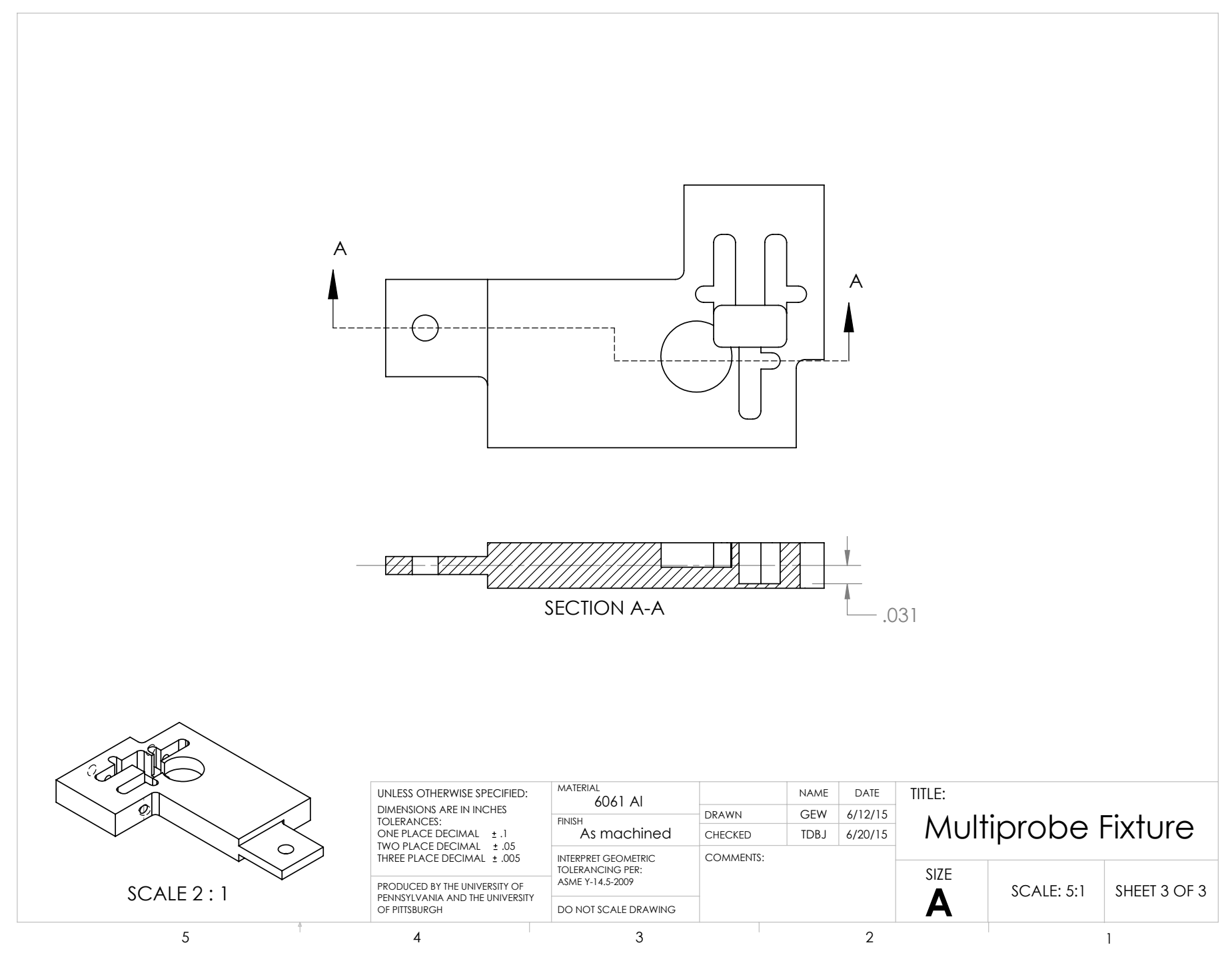




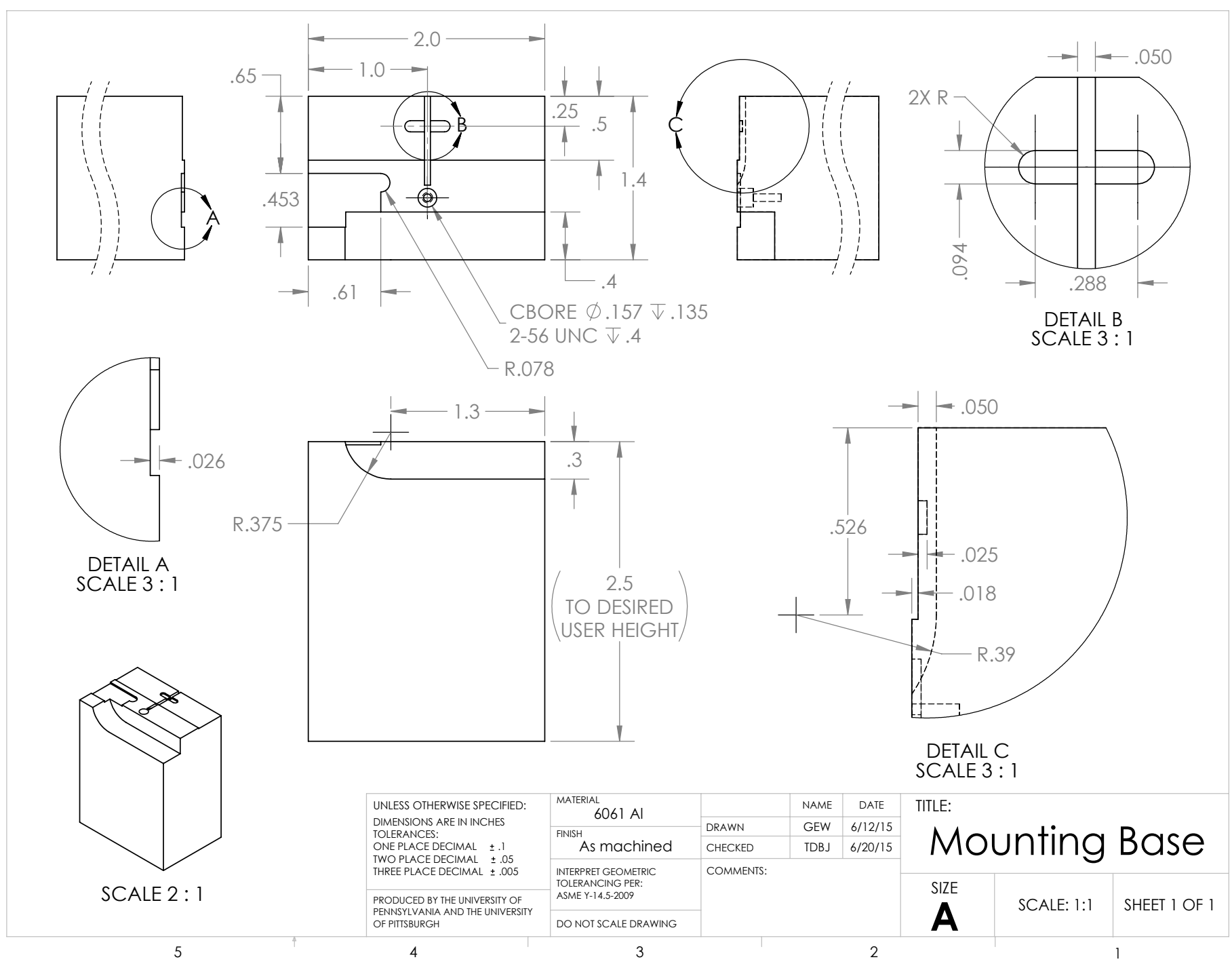




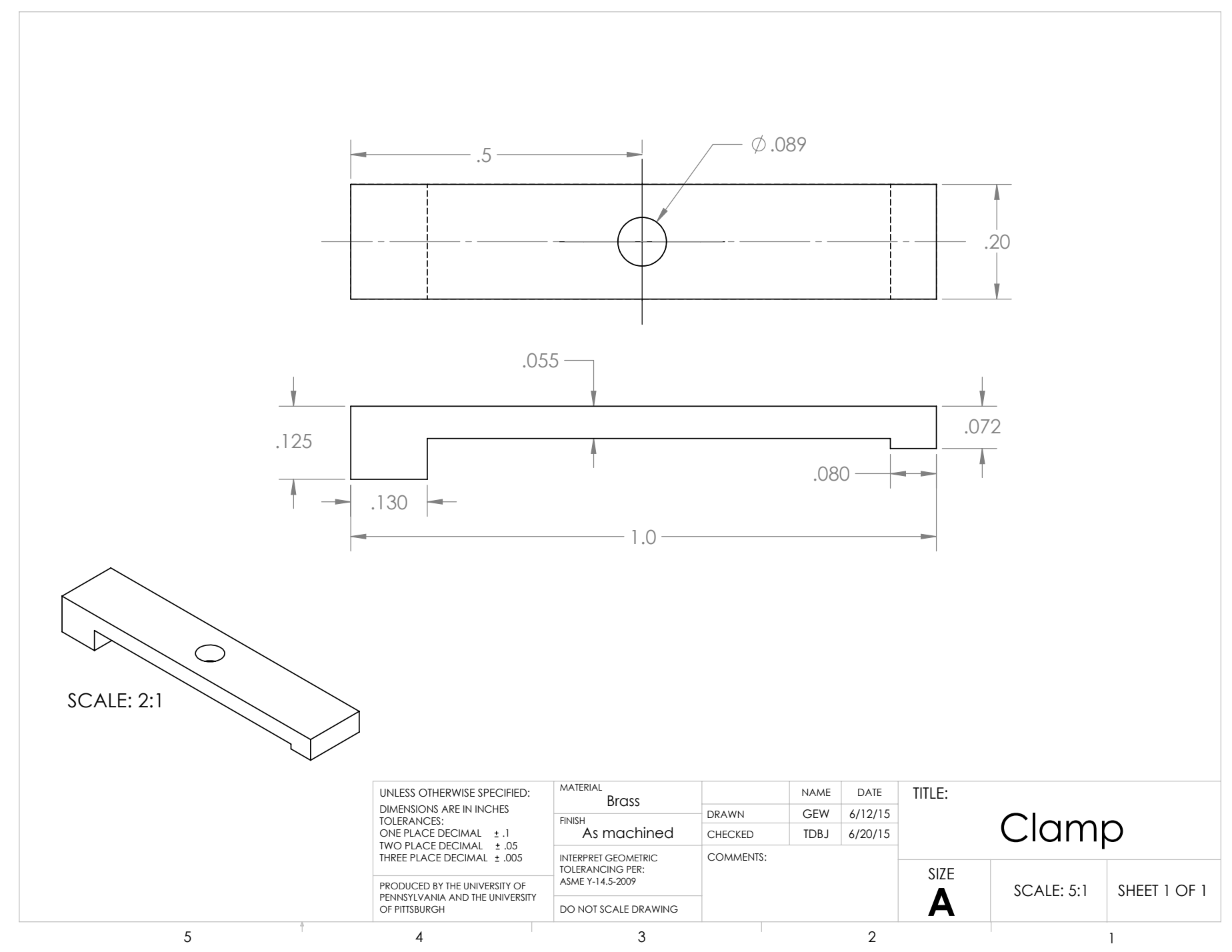




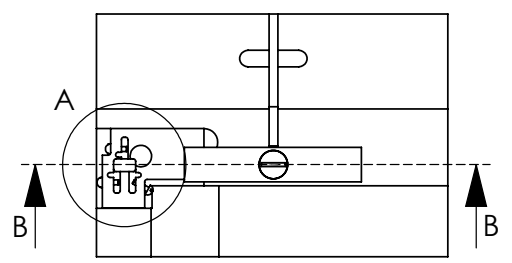

\begin{tabular}{|c|l|c|}
\hline ITEM NO. & \multicolumn{1}{|c|}{ PART NAME / DESCRIPTION } & QTY. \\
\hline 1 & Multiprobe fixture & 1 \\
\hline 2 & Mounting base & 1 \\
\hline 3 & Clamp & 1 \\
\hline 4 & Spring & 1 \\
\hline 5 & $\# 2-56$ slotted round head fastener & 1 \\
\hline 6 & $\begin{array}{l}\text { \#000-120 brass, slotted round head } \\
\text { fastener }\end{array}$ & 3 \\
\hline
\end{tabular}

2
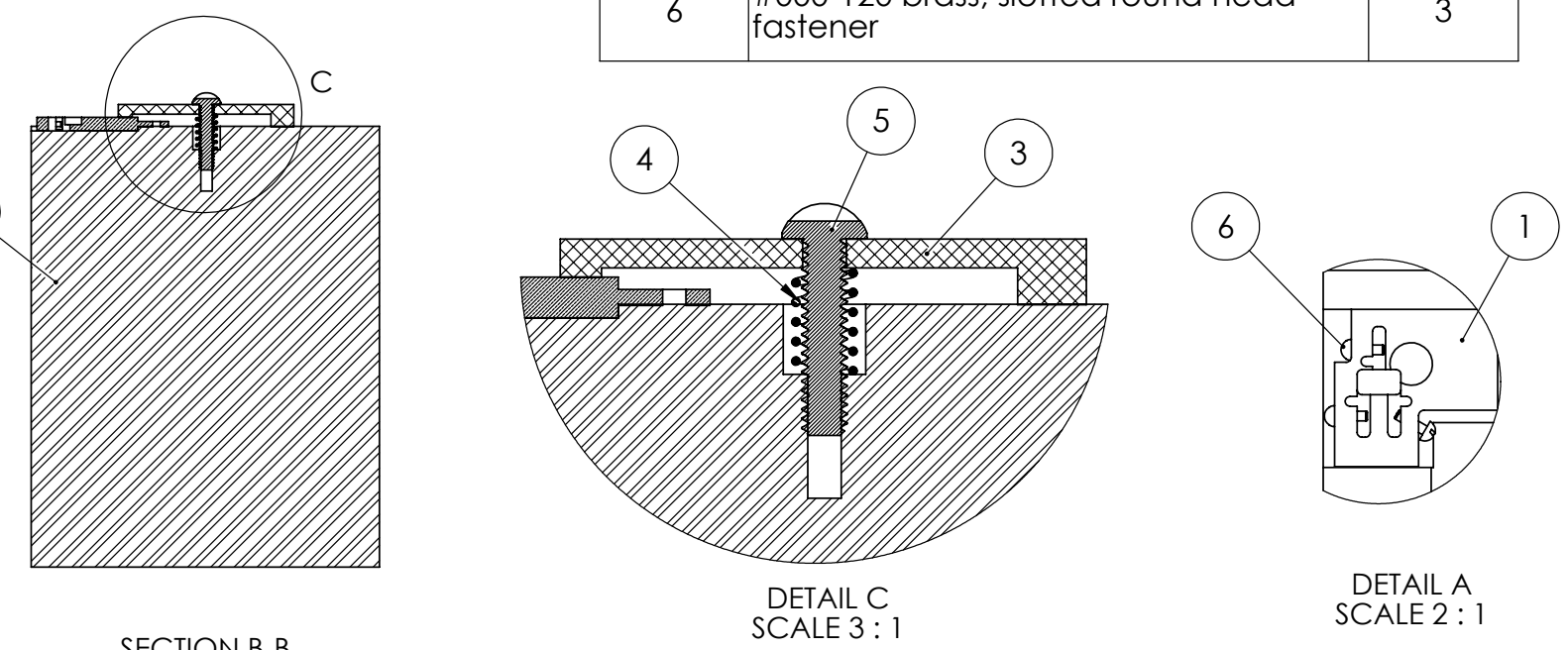

DETAIL A
SCALE $2: 1$

SECTION B-B

SCALE 3:

Notes:

PRODUCED BY THE UNIERSITY OF PENSSYVANAA AND THE

INTERRET GEOMETRR
TOLERANCING PER:

DO NOT SCALE DRAWING

3
1) Select any reasonable spring (part 4) that fits the

mounting base counterboreand clamp fastener

2) More modern fastener sizes may be substituted

for the \#000-120 fasteners

NAME DATE $\quad$ TITLE:

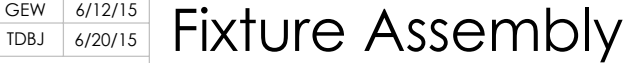

3) Sand / round the tip of the \#000-120 or

equivalent fasteners to avoid stress concentrations at the fastener-sample interface \begin{tabular}{l|l|l}
\hline SIZE & SCAle: 1:1 & SheEt 1 OF 1 \\
\hline
\end{tabular} 